\title{
e coût du système immunitaire : un facteur important de la sélection naturelle
}

La notion de «coût» représente un important concept de la théorie moderne de l'évolution. Si les fonctions physiologiques n'étaient pas coûteuses, les organismes vivants auraient tous des traits d'histoire de vie similaires (croissance rapide, durée de vie et fécondité importantes). Or, ce n'est pas le cas. En effet, les organismes ne possèdent qu'une quantité limitée de ressources qu'ils doivent répartir entre leurs diverses fonctions afin de maximiser leur valeur sélective ou «fitness» (contribution à la génération suivante). II existe donc dans les organismes un compromis d'allocation de ressources (nutriments et énergie) entre les différentes fonctions consommatrices, phénomène appelé tradeoff par les Anglo-Saxons [1]. Traditionnellement, seuls les principaux traits d'histoire de vie des organismes, comme la survie, la croissance et la reproduction, étaient pris en compte dans l'étude de ces tradeoff. Des travaux récents ont montré l'importance de la fonction immunitaire dans les phénomènes d'allocation de ressources, et donc dans l'étude de l'évolution des traits d'histoire de vie.

La majorité des êtres vivants sont soumis à l'influence de divers parasites qui exploitent les ressources de leurs hôtes pour assurer leur propre reproduction. Ceci se fait bien souvent aux dépens de la survie et de la fécondité de l'hôte. Face à cette menace constante, la sélection naturelle a favorisé chez les hôtes le développement d'une très large gamme d'adaptations permettant de réduire les effets indésirables des parasites. Parmi ces adaptations, le système immunitaire tient une place majeure.
I'hypothèse selon laquelle l'évolution de l'immunité pourrait se réaliser aux dépens d'autres fonctions importantes de l'hôte [2, 3] a été émise. En effet, l'activation et le maintien du système immunitaire sont supposés avoir un coût énergétique qui doit entrer en compétition avec celui d'autres fonctions demandeuses [4, 5]. Ainsi, certains des effets négatifs attribués aux infections parasitaires résulteraient moins des dommages directs dus au parasite lui-même que du coût imposé par l'activation du système immunitaire de l'hôte. Cependant, si un tel coût existe, on peut s'attendre à ce qu'il ne soit pas toujours décelable dans la nature. D'une part, le coût de la défense immunitaire contre une infection parasitaire peut être confondu avec celui du parasitisme (l'action du parasite et la réaction du système immunitaire sont concomitants). D'autre part, les organismes sont capables de compenser des demandes énergétiques accrues en augmentant l'acquisition de ressources, par exemple leur prise de nourriture. Dans ce dernier cas, le coût serait masqué, bien que l'hôte paye un prix pour prévenir l'établissement et la propagation du parasite. De récents travaux sur des insectes ont démontré de façon directe qu'il existe un coût immédiat à l'activation de la fonction immunitaire [6]. Le système immunitaire des insectes, comme celui de tous les invertébrés, développe une réponse de type immunité innée. Les hémocytes détruisent les agents pathogènes par phagocytose ou par libération de molécules solubles toxiques pour les parasites. II est possible d'obtenir de telles réponses immunitaires en utilisant des micro-billes de latex ou des lipopolyaccharides (LPS) extraits $d^{\prime} E$. coli. Ces immunogènes n'étant ni vivants ni pathogènes, tout effet négatif direct du parasitisme est exclu, et I'on peut donc évaluer le coût métabolique de la seule réponse immunitaire. Une telle réponse, induite contre ces immunogènes injectés à des bourdons (Bombus terrestris) totalement privés de nourriture, est associée à une réduction de leur survie de $50 \%$ à $70 \%$ par rapport à des insectes témoins qui n'ont reçu qu'une injection de liquide physiologique. En revanche, si les bourdons sont nourris avec de l'eau sucrée, aucune différence de survie n'est observée entre les deux groupes [6]. Comme chez les invertébrés, la réponse immunitaire des vertébrés est associée à un coût sur leur valeur sélective. En plus d'une immunité innée, les vertébrés possèdent une immunité acquise, reposant sur de nombreuses cellules portant une très large gamme de récepteurs capables de reconnaître un nombre quasiinfini d'antigènes. En vaccinant des gobe-mouches sauvages (Ficedula hypoleuca) contre la diphtérie et le tétanos, une équipe fino-suédoise a pu observer, chez cet oiseau, une réduction de l'effort de reproduction associée au développement de la réponse immunitaire [7]. En effet, les oiseaux vaccinés (mais non soumis aux pathogènes) nourrissent moins leurs petits que les oiseaux non vaccinés. Par conséquent, les poussins produits par les oiseaux vaccinés sont moins nombreux et plus petits que ceux des oiseaux non vaccinés.

Dans le cas des oiseaux comme dans celui des bourdons, le coût de la fonction immunitaire n'est mis en évidence que dans des conditions de 
stress. Les bourdons étaient en effet privés de nourriture, et les oiseaux étaient en période de reproduction, période durant laquelle ils dépensent beaucoup d'énergie à nourrir les jeunes et passent peu de temps à se nourrir eux-mêmes. Dans ces conditions, l'activation de la réponse immunitaire nécessite donc une quantité d'énergie excessive qui doit être prise sur le quota alloué à d'autres fonctions comme la survie ou la reproduction. Ce coût passe en revanche inaperçu lorsque les organismes disposent d'assez de ressources pour le compenser. Dans les conditions naturelles, l'accès aux ressources se fait aussi au prix de nombreux efforts. II semble donc indispensable qu'un organisme qui maintient et utilise une machinerie immunitaire très coûteuse soit aussi capable de minimiser le prix de son accès aux ressources, sous peine de subir une contre sélection.

En raison du coût du système immunitaire, la sélection naturelle ne devrait favoriser une immunocompétence élevée que lorsque celle-ci est bénéfique. Ainsi, l'allocation des ressources à la fonction immunitaire des organismes devrait être plus importante lorsqu'ils sont exposés à un plus grand nombre de parasites et de pathogènes [8]. Ceci expliquerait la variabilité naturelle de la résistance des organismes aux parasites. C'est ce que tendraient à montrer les corrélations observées par l'étude de Nunn et al. [9] chez des primates supposés être plus ou moins exposés à des maladies sexuellement transmissibles. Cette étude a montré que le nombre de leucocytes, quel que soit le type de cellules (neutrophiles, lymphocytes et monocytes), était plus important chez les espèces polygames que chez les espèces monogames. Cependant, ces corrélations ne permettent pas d'affirmer avec certitude, pour le moment, que c'est bien le risque d'infection par des maladies sexuellement transmissibles qui est directement responsable de la différence d'allocation à la fonction immunitaire.

De nombreux programmes de recherches appliqués à la lutte contre les parasites et les maladies infectieuses se fondent sur une stratégie consistant à élever le niveau de résistance ou d'immunocompétence des animaux et des hommes. Or, le coût de la réponse immunitaire devrait imposer des contreparties (réduction de la croissance, fécondité ou survie). Par exemple, il paraît intéressant d'augmenter la résistance des vecteurs de maladies parasitaires (insectes principalement) vis-à-vis des agents infectieux qu'ils transmettent. Cependant, du fait du coût de cette résistance, seuls les insectes non résistants seront sélectionnés et resteront dans la population. De même, s'il semble potentiellement avantageux pour les éleveurs d'augmenter la résistance des animaux domestiques, ils devront en contrepartie augmenter les apports d'aliments à leurs animaux pour compenser le coût physiologique qui en résulterait. Les recherches menées sur les vaccins visent aussi à augmenter les défenses immunitaires humaines pour mieux résister aux maladies. II serait alors indispensable, lors de la vaccination d'enfants en bas âge, de maintenir une bonne alimentation afin d'éviter d'affecter leur croissance. Par conséquent, si le coût de la fonction immunitaire est important et répandu, le succès de cette stratégie d'élévation du niveau de résistance risque d'être compromis si le nécessaire n'est pas fait pour en compenser le coût.

1. Stearns SC. The Evolution of Life Histories. Oxford: Oxford University Press, 1992

2. Sheldon BC, Verhulst S. Ecological immunology: costly parasite defences and trade-offs in evolutionary ecology. Trends in Ecology and Evolution 1996; 11: 317-21.

3. Kraaijeveld AR, Godfray HCJ. Trade-off between parasitoid resistance and larval competitive ability in Drosophila melanogaster. Nature 1997; 389: 278-80.

4. Nordling D, Andersson M, Zohari S, Gustafsson L. Reproductive effort reduces specific immune response and parasite resistance. Proc Roy Soc 1998 ; $265: 1291-8$.

5. Koenig C, Schmid Hempel P. Foraging activity and immunocompetence in workers of the bumble bee, Bombus terrestris L. Proc Roy Soc 1995; 260 : 225-7.

6. Moret $Y$, Schmid-H empel P. Survival for immunity: the price of immune system activation for bumblebee workers. Science 2000; 290: 1166-8.

7. Ilmonen $P, T$ aarna $T$, Hasselquist $D$. Experimentally activated immune defence in female pied flycatchers results in reduced breeding success. Proc Roy Soc 2000; 267: 665-70.

8. Jokela J, Schmid-H empel P, Rigby MC. Dr. Pangloss restrained by the Red Queen - steps towards a unified defence theory. 0ikos 2000; 89: 267-78. 9. Nunn CL, Gittleman JL, Antonovics J. Promiscuity and the primate immune system. Science $2000 ; 290$ : 1168-70.

\section{Yannick Moret}

Eidgen össische Technische $\mathrm{H}$ ochschule (ETH) Zürich, Experimental Ecology, ETH-Zentrum, NW, CH -8092 Zürich, Suisse 